\title{
Case Report: Amyloidosis of Colon Presented as A Cat Scratch Colon
}

\author{
Yue Guan ${ }^{1}$, Yu Dong ${ }^{1}$, Yijun $\mathrm{Xu}^{1}$, and Jie Yuan ${ }^{1}$ \\ ${ }^{1}$ Nanjing First Hospital
}

November 25, 2021

\begin{abstract}
Endoscopic features of gastrointestinal amyloidosis can be nonspecific. In our case, it presented as cat scratch colon, which led to a misdiagnosis as collagen colitis.

Case Report: Amyloidosis of Colon Presented as A Cat Scratch Colon

Yue Guan $^{1}$, Yu Dong ${ }^{1}$, Yijun $\mathrm{Xu}^{1}$

Department of Gastroenterology, Nanjing First hospital, Nanjing Medical university

Correspondence: Jie Yuan, Department of Gastroenterology, Nanjing First hospital, Nanjing Medical university. Email: jim0790@sina.com

I confirm that written patient consent has been signed and collected in accordance with the journal's patient consent policy.

Abstract: Endoscopic features of gastrointestinal amyloidosis can be nonspecific. In our case, it presented as cat scratch colon, which led to a misdiagnosis as collagen colitis.

Introduction: Systematic amyloidosis is characterized by the extracellular deposition of an abnormal fibrillar protein in various organs, including GI tract. Clinical manifestations can vary from proteinuria or renal impairment, congestive cardiac failure, damage of autonomic nervous system, to gastrointestinal dysfunction. These all depend on the type of on the precursor protein that forms the amyloid fibril11Baker K R, Rice L. The amyloidoses: clinical features, diagnosis and treatment[J]. Methodist DeBakey cardiovascular journal, 2012, 8(3): 3.. Endoscopic manifestations of systematic amyloidosis in GI tract are nonspecific, such as polys or mucosal erosion22Sattianayagam P T, Hawkins P N, Gillmore J D. Systemic amyloidosis and the gastrointestinal tract[J]. Nature reviews Gastroenterology \& hepatology, 2009, 6(10): 608-617., but seldom can it be cat scratch colon, which is a sign indicated collagen colitis. We present a case of an elderly lady with amyloidosis of colon presented as cat scratch colon, which was misdiagnosed as collagen colitis.
\end{abstract}

Case report

A 72-year-old female was admitted to Gastroenterology Department, Nanjing First Hospital in April 2020 to investigate chronic diarrhea. She had had watery diarrhea 3-4 times a day for about 30 days. Nothing significant was detected on physical examination. Blood results and urinalysis were unremarkable except for mild hypoproteinemia and hypoalbuminemia (Total Protein: $59.30 \mathrm{~g} / \mathrm{L}, \mathrm{RI}: 65-85 \mathrm{~g} / \mathrm{L}$; Albumin: $20.70 \mathrm{~g} / \mathrm{L}$, RI: $40-55 \mathrm{~g} / \mathrm{L}$ ). Fecal analysis was negative for common endoparasites and fungi. To further investigate the disease, we performed colonoscopy on 22th April 2020. 
At the ascending colon, several bright red linear marks were spotted, which resembled cat scratch sign. The remainder of the colorectal mucosa was macroscopically normal. A tentative diagnosis of collagenous colitis was made at that time. Two biopsies were taken from the cat scratch site and rectum. The pathologist reported that there seemed to be collagen deposition in the lamina propria.

We treated this patient as collagenous colitis, prescribing her probiotics(Bifid Lriple $630 \mathrm{mg}$ bid)and budesonide( $9 \mathrm{mg}$ qd). After 7 days of treatment in hospital, the frequency of diarrhea was somewhat reduced, so the patient was discharged. In our 3-month follow-up session, she still reported diarrheal but no further abnormalities.

In Aug 2020, the patient represented herself to us with edema of both lower limbs, fatigue and weight loss. She also mentioned that there had been ecchymoses on her abdominal skin in the past 3 months. We repeated standard blood tests, urinalysis, fecal analysis. This time her laboratory tests showed compromised kidney function (Cr $132 \mathrm{umol} / \mathrm{L}$, Urea $10.66 \mathrm{mmol} / \mathrm{L}, 24 \mathrm{~h}$ Urine protein $1.78 \mathrm{~g}$ ), hypoproteinemia (Alb $21.25 \mathrm{~g} / \mathrm{L}$ ), and anemia ( $\mathrm{Hb} 103 \mathrm{~g} / \mathrm{L}$ ). We suspected that she had multiple organ dysfunction, which could be caused by systematic amyloidosis. So, we sent the previous biopsy samples to the pathology lab again for second opinion. This time, the pathologists found Congo red stained amyloid in the submucosal of the colon, and apple green birefringence under cross-polarized light. (Figure 1)

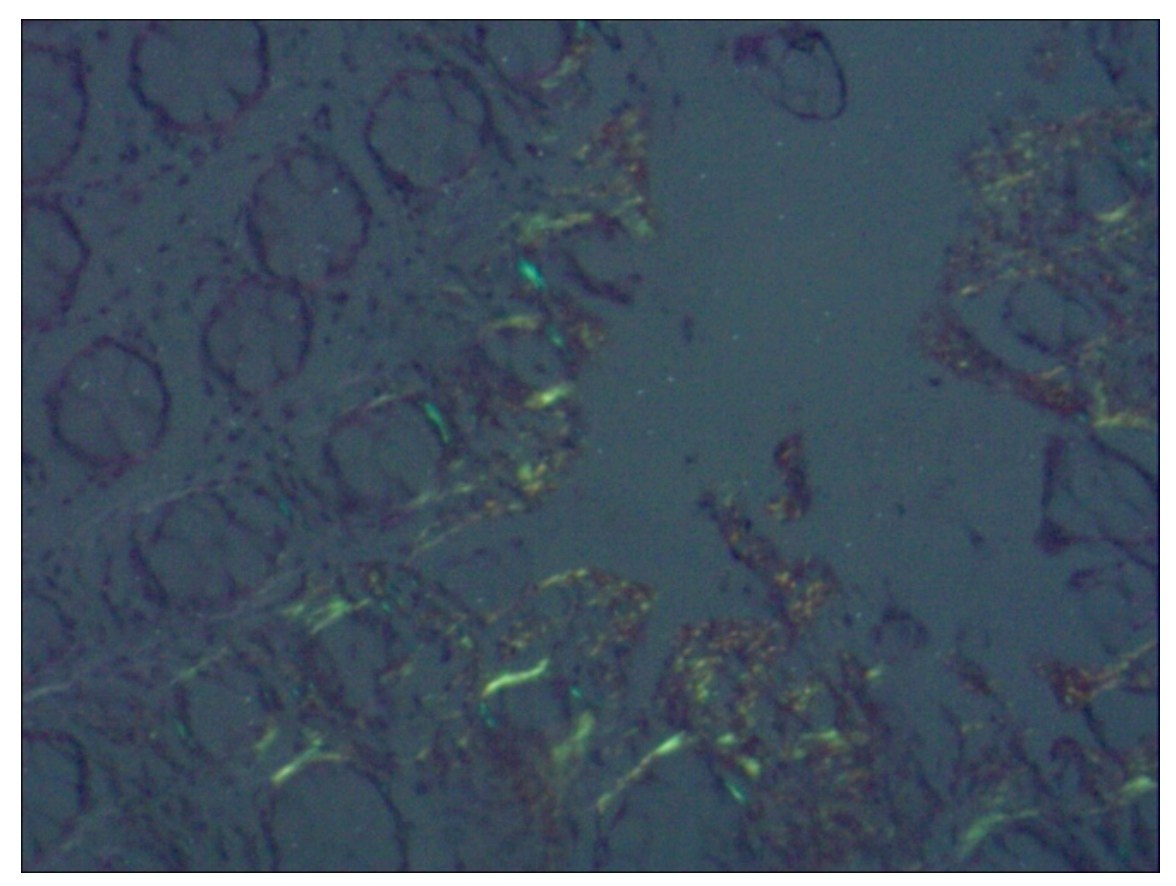

Figure 1 cross-polarized light x200

Follow-up tests were performed to evaluate her condition. Echocardiography showed slightly thickened interventricular septum(IVSD 12mm). Quantitation of serum free light chains revealed $x$ light chain is $3.41 \mathrm{~g} / \mathrm{L}$, while $\lambda$ light chain is $5.07 \mathrm{~g} / \mathrm{L}, \chi / \lambda 0.67$. The urine free light chains $\lambda$ level also increased $(111.0 \mathrm{mg} / \mathrm{L}), \mathrm{F} \chi / \mathrm{F}$ $\lambda$ is 0.35 . Bone marrow biopsy revealed abnormal plasma cells which include CD19(+),CD38(+),CD56(+), CD138(+), cLambda(+). As a result, this patient was diagnosed of AL amyloidosis, and later received chemotherapy of bortezomib $1.8 \mathrm{mg}$ on $\mathrm{d} 1, \mathrm{~d} 4, \mathrm{~d} 8$, d11. She was in better condition when discharged, sadly we found out she passed away 10 months later because of renal failure.

Discussion

Systemic amyloidosis is characterized by the extracellular deposition of protein in an abnormal fibrillar form. 
Endoscopic findings of systemic amyloidosis can be found in all digestive tracts, and the manifestation varies from mucosal congestion, erosion, ulcer, nodular protuberance to polypoid changes11Hokama A, Kishimoto K, Nakamoto M, et al. Endoscopic and histopathological features of gastrointestinal amyloidosis. World $J$ Gastrointest Endosc . 2011;3(8):157-61.. It has been reported that in AL amyloidosis, the most common endoscopic presentation is polypoid protrusions because amyloid mostly deposits in muscularis mucosae, submucosa and muscularis propria, whereas in AA amyloidosis, amyloid mostly deposits in propria mucosae mucosal, which leads to friability and erosions22Tada, S., Iida, M., Yao, T., et al. (1994). Endoscopic features in amyloidosis of the small intestine: clinical and morphologic differences between chemical types of amyloid protein. Gastrointestinal endoscopy , 40 (1), 45-50.. But there has been no report of cat scratch colon in amyloidosis patient in current literature.

Cat scratch colon is a rare colonoscopic finding, first described in 2007 with a prevalence of $0.25 \%$. It is defined as bright, erythematous linear breaks that arise spontaneously in cecum and ascending colon33McDonnell W, Loura FC, Pointon MJ, et al. Cat scratch colon. Gastrointestinal Endoscopy . 2007;65(5):AB268. . The mechanism of cat scratch sign remains unclear, but it can be related to both barotrauma from air insufflation and lack of compliance of bowel wall44Park EJ, Lee JS, Lee TH,, et al. "Cat scratch colon" in a patient with ischemic colitis. Clin Endosc . 2015;48(2):178-80..It is often seen in patients of collagenous colitis, which is why we made the diagnosis of collagenous colitis in the first place. Both amyloidosis and collagenous colitis can present as deposition of thickened subepithelial band of eosinophilic-hyaline substance. Amyloidosis of the rectum was reported of mimicking collagenous colitis in the past, therefore, we suggest that congo red stain should be considered in pathological examination for samples presented with histologic changes of collagenous colitis, especially when patient has multi-system dysfunction55García-González R, Fernández FA, Francisca Garijo M, et al. Amyloidosis of the Rectum Mimicking Collagenous Colitis. Pathology-Research and Practice.1998;194(10):731-5.. Cat scratch colon can also be found in patient with ischemic colitis ${ }^{\mathrm{iv}}$ and chronic cholestasis66Purnak T, Ozaslan E, Yildiz A, et al. The cat scratch colon sign in a patient with chronic cholestasis.Endoscopy . 2010;42 Suppl 2:E117., which can be because these diseases damaged the Integrity of large intestine wall.

Gastrointestinal symptoms are very common in patients witch systematic amyloidosis. Colon endoscopy and biopsy are very important diagnostic tools for this disease. Due to the deposition of proteins in colon wall, we believe it is also the reason why amyloidosis can present as cat scratch colon. Doctors should be aware of this possibility and take biopsy from nearby area if possible. As treatment of amyloidosis can include glucocorticoid, it can explain the temporary relief of our patient. Also, since pneumoperitoneum after the occurrence of cat scratch colon was reported, endoscopists should be careful with air insufflation during the procedure because pneumoperitoneum can happen secondary to cat scratch colon77Diaz-Sanchez A, Riesco JM, Forero A,et al. Pneumoperitoneum due to cat scratch colon. Is it really such an innocent disease? Dig Liver Dis . 2015;47(6):527-8.

Authors' contributions

Yue Guan. drafted the manuscript and analyzed and interpreted the data.

Yu Dong and YiJun Xu collected clinical information when the patient was first admitted.

Jie Yuan. conceived and designed the project. All authors read and approved the final manuscript.

Funding: Not applicable.

Acknowledgements: The patient provided written permission for publication of this case report. All data generated during this study are included in this published article and its supplementary information files. The study was approved by Nanjing First Hospital. 


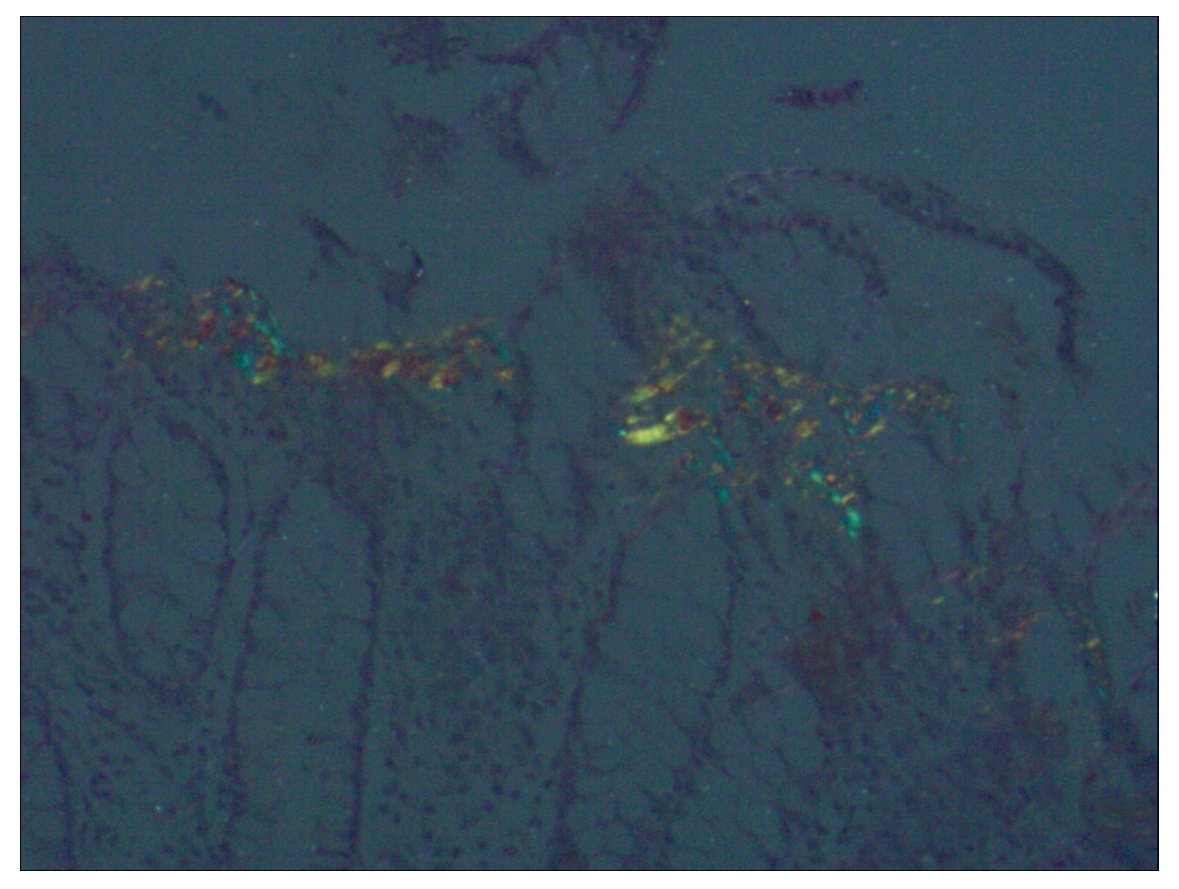

